\title{
Rethinking the input: Skewed distributions of exemplars result in broad generalization in category learning
}

\author{
Paulo F. Carvalho ${ }^{1}$, Chi-hsin $\mathrm{Chen}^{2}$, \& Chen $\mathrm{Yu}^{3}$ \\ ${ }^{1}$ Carnegie Mellon University, ${ }^{2}$ The Ohio State University, ${ }^{3}$ Indiana University
}

\begin{abstract}
Author Note
Paulo F. Carvalho, Human-Computer Interaction Institute, Carnegie Mellon University; Chi-hsin Chen, Department of Otolaryngology-Head and Neck Surgery, The Ohio State University; Chen Yu, Department of Psychological and Brain Sciences and Cognitive Science Program, Indiana University.

The first two authors contributed equally to this work and share first-authorship. Contact: Paulo F. Carvalho, Human-Computer Interaction Institute, Carnegie Mellon University, Newell-Simon Hall, 5000 Forbes Ave., Pittsburgh, PA, 15213; pcarvalh@cs.cmu.edu or Chi-hsin Chen, Department of Otolaryngology-Head and Neck Surgery, The Ohio State University, 915 Olentangy River Road, Columbus, OH, 43212; chi-hsin.chen@osumc.edu.
\end{abstract}




\begin{abstract}
What we learn about the world is affected by the input we receive. Many extant category learning studies use uniform distributions as input in which each exemplar in a category is presented the same number of times. Another common assumption on input used in previous studies is that exemplars from the same category form a roughly normal distribution. However, recent corpus studies suggest that real-world category input tends to be organized around skewed distributions. We conducted three experiments to examine the effects of skewed input distributions on category learning and generalization. Across all studies, skewed input distributions resulted in broader generalization than uniform and normal distributions. Our results not only suggest that the current category learning theories may underestimate category generalization but also challenge the current theories to explain category learning in the real world with skewed, instead of the normal or uniform distributions often used in experimental studies.
\end{abstract}

Keywords: input distributions; skewed distributions; category learning; generalization 
Rethinking the input: Skewed distributions of exemplars result in broad generalization in category learning

What we learn about the world is constrained by the input we get (Kruschke, 2003; Smith, Colunga, \& Yoshida, 2010). For example, children's initial visual and auditory input creates the basis for what they can learn (Werker, Yeung, \& Yoshida, 2012). A characteristic of the input that is often overlooked when studying learning is the distribution of the exemplars in the input, that is, how often each exemplar is seen relative to other exemplars in the same group. In fact, most learning studies equate the number of times each object is seen, guaranteeing a uniform distribution from which each exemplar contributes equally to category learning (Goldstone, Kersten, \& Carvalho, 2017). However, the type of distributions we use in the lab is very different from the real-world input distributions. Contrary to most laboratory settings, real-world input seems to be organized around skewed distributions (Bambach, Crandall, Smith, \& Yu, 2018; Boyd \& Goldberg, 2009; Clerkin, Hart, Rehg, Yu, \& Smith, 2017; Smith, Jayaraman, Clerkin, \& Yu, 2018; Smith \& Slone, 2017) — those in which a few items have a much higher likelihood of being experienced, with a long tail of items that are experienced much less frequently (see Figure 1A). For example, a child learns about a category representation of dog based on many exposures to her own dog encountered in everyday contexts along with fewer exposures of many other dogs encountered on other occasions.

This is an important issue because the cognitive processes involved in learning are likely to be dependent on the type of input that the system gets (Smith et al., 2010, Smith et al. 2018). Thus, if most of early human experience involves skewed input distributions, it is likely that our 
current theories about learning mechanisms based on normal or uniform input distributions might not closely approximate how people learn categories in the real world. This is equivalent to developing a mechanism around learning to swim with only practice from land simulated swimming — it fails to take into the account the friction and buoyancy created by the water itself. In this paper, we investigate the impact of skewed input distributions (the water) on category learning and generalization (the swimming).

How might the input distribution influence what is learned in a visual category learning task? Previous research has suggested that learners represent their experience as a unimodal —approximately normal— distribution (Flannagan, Fried, \& Holyoak, 1986). That is, even when exposed to another distribution type, such as a uniform distribution of exemplars during a category learning task, learners still represent the category as a normal distribution in the same space (Duffy, Huttenlocher, Hedges, \& Crawford, 2010; Flannagan et al., 1986; Huttenlocher, Hedges, \& Vevea, 2000; but see Duffy \& Smith, 2018). Moreover, this "central bias" is hard to change, requiring extensive experience for learners to change their representations to a multimodal distribution (Huttenlocher et al., 2000). Thus, input organized in a skewed distribution could influence the properties (e.g., the mean, or the peak of the normal distribution, and the variance, or the range of items included in the normal distribution) of the resulting representation the learner creates (Flannagan et al., 1986). For example, if learners assume a normal distribution centered around the most frequent item of a given category, but that category has a skewed distribution (see Figure 1A), the representation they create will be wider than the equivalent representation created from a normally-distributed category (see Figure 1B). This is 
because learners are creating a normally distributed representation that encompasses all stimuli observed but is centered around the most frequent item.

(A)

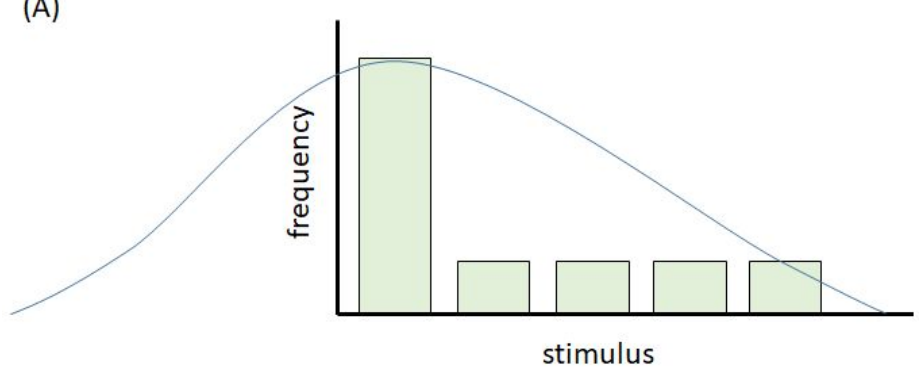

(B)

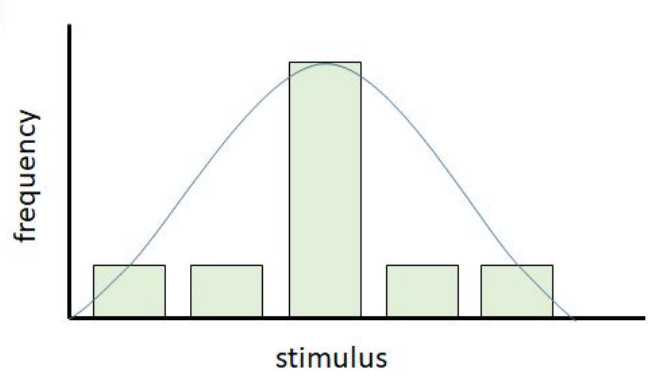

Figure 1: Representations of (A) a right-skewed distribution and (B) a normal distribution with the same total number of exemplars. The blue line represents predicted generalization gradients resulting from experience with each type of distribution.

Another relevant finding from the literature is that participants are sensitive to the variance in the exemplars properties (Fried \& Holyoak, 1984; Cohen et al., 2001; Sakamoto et al., 2008). When exposed to two categories with unequal variance, participants tend to categorize novel items into the category with the larger variance (Fried \& Holyoak, 1984). The hypothesis is that when learning the categories learners estimate category variability by sequentially comparing items and later using this estimated mean and variation to classify novel items (Sakamoto et al., 2008). Therefore, categories with higher variance will be wider, because the estimated mean and variation will be larger.

According to this hypothesis, distributions with different skewness will have an impact on the variance of the stimuli presented. If learners do not take into account the overall real 
variance and mean of the distributions but instead use perceived values derived from sequential comparisons, the skewed distribution will be perceived as having higher variance because the distance between the most frequent item and the least frequent item will be larger than in the case of a normal distribution. For example, the input distribution represented in Fig.1 (A) has higher variance than the one represented in Fig. 1 (B) because the distance between the more frequent item and the most extreme item is larger in (A) than in (B).

Taken together, both the possibility that learners represent seen items as a normal distribution centered around the most frequent item or estimate variance and mean based on sequential comparisons suggest that the input distribution will have an impact on category representations and influence how broadly participants generalize their learning. To test whether skewed input distributions would result in wider category representations, as predicted above, we designed three studies.

In the first study, we taught participants two categories of novel objects, one using a uniform input distribution and another using one of three unimodal input distributions: right-skewed, normal, or left-skewed distributions. We used a uniform distribution for the second category to keep constant the properties of the second category and guarantee that any difference found was due to the characteristics of the unimodal distribution only. Following category learning, we compared learners' generalization responses resulting from experience with the different unimodal distributions. In Experiment 2, we tested whether other properties of the distributions (the steepness of the distributions) affected learners' generalization patterns. Finally, in Experiment 3 we directly compared generalization from training data generated from 
a single distribution, either skewed or normal, that did not require the inclusion of a second category.

\section{Experiment 1}

\section{Method}

Participants. We used G*Power (Faul, Erdfelder, Lang, Buchner, 2007) to calculate the required sample size to detect a small effect size $(f=0.15)$ for the within-subject effect of type of input distribution, considering 2 between-subject groups (given counterbalancing conditions, see details on procedure below) and 3 levels of within-subject measurement, with $\alpha=.05$. The sample size required to achieve $75 \%$ power was 66 participants. Because of the limited previous research to provide a precise estimate of expected effect size, we decided to collect data from more than the minimum number of participants described above, approximately 80 participants to accommodate for potential dropout. We did not analyze the data before completing data collection with the target sample size.

84 volunteer undergraduate students at Indiana University (48 females, mean age: 19) participated in this study in return for course credit. However, due to technical problems, 3 participants did not finish the experiment and were excluded from the analyses. 81 participants completed all conditions. Data from one participant was excluded from analyses due to not following instructions (responding with the same key to all test trials). Recruitment and experimental procedures were approved by Indiana University's Institutional Review Board and all participants gave informed consent prior to participation. 
Design and Procedure. The stimuli were sets of novel-looking flowers, each containing 21 flowers; there were 3 different sets, all with the same properties but different visual appearance. Objects in each set varied along a continuum in one perceptual feature (shape of the disk, the color of petals, the length of the stem). Take Fig. 2A as an example, the flower on the left extreme has a diamond-shaped disk whereas the flower on the right extreme has a circle-shaped disk. The training items were the 5 most extreme items on each side (e.g., items 1-5 with a more diamond-like shape and items 17-21 with a more circle-like shape).

There were two training categories, one with a uniform distribution and the other with a unimodal distribution (Fig. 2B). There were 3 unimodal distribution conditions, a right-skewed distribution with a peak at the exterior of the continuum, a normal distribution with a peak in the middle, and a left-skewed distribution with a peak in the interior of the continuum. For each set, which side (items 1-5 or 17-21) was the uniform or unimodal distribution was counterbalanced across participants. The members in each category occurred 60 times in total. Members in the uniform distribution each occurred 12 times. One member in each of the skewed distributions occurred 40 times while the other members occurred 5 times each. 
(A) Training Categories: Experiments $1 \& 2$

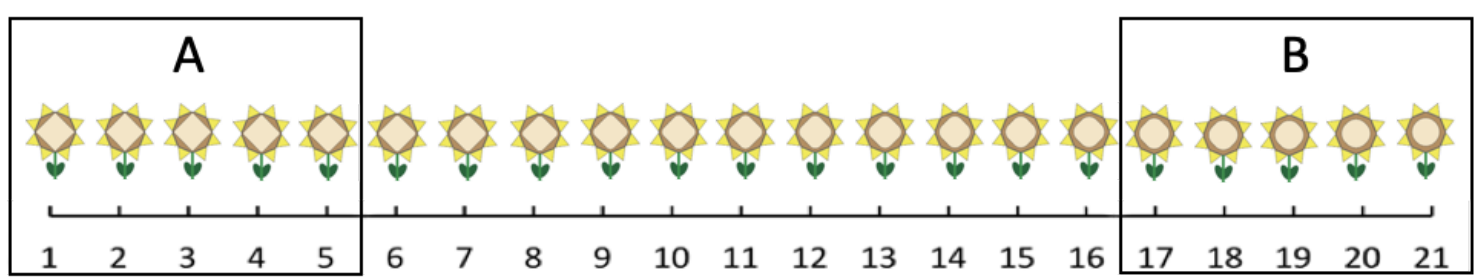

(B) Category Distributions: Experiment 1

Right Skewed

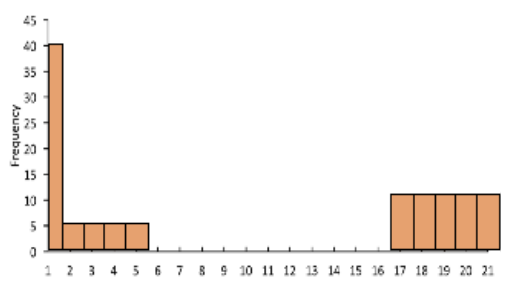

Normal

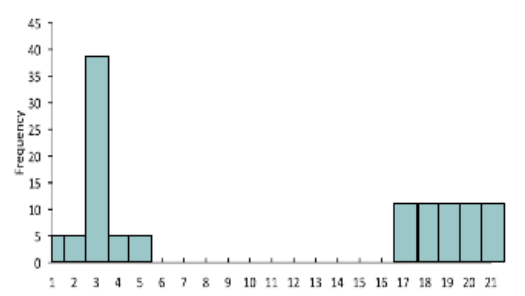

Left Skewed

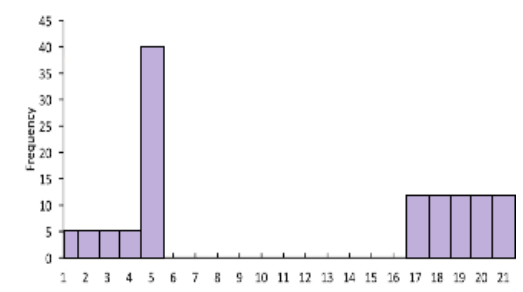

(C) Training Category: Experiment 3

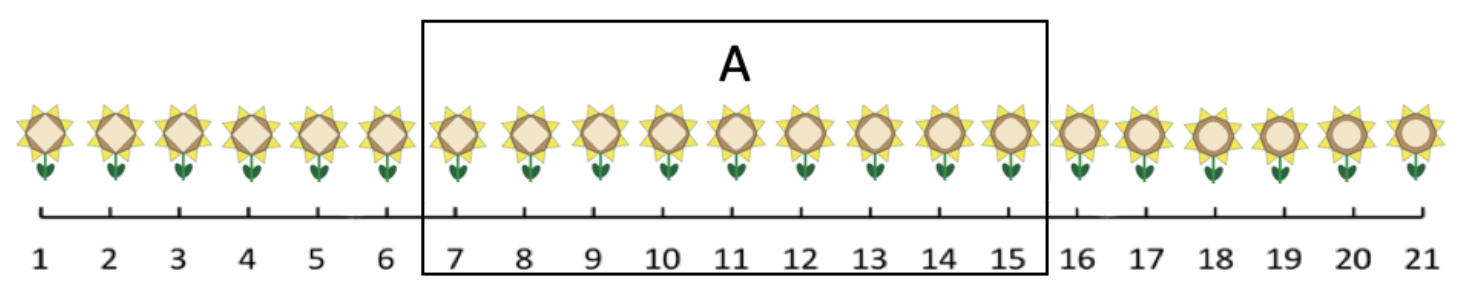

(D) Category Distributions: Experiment 3
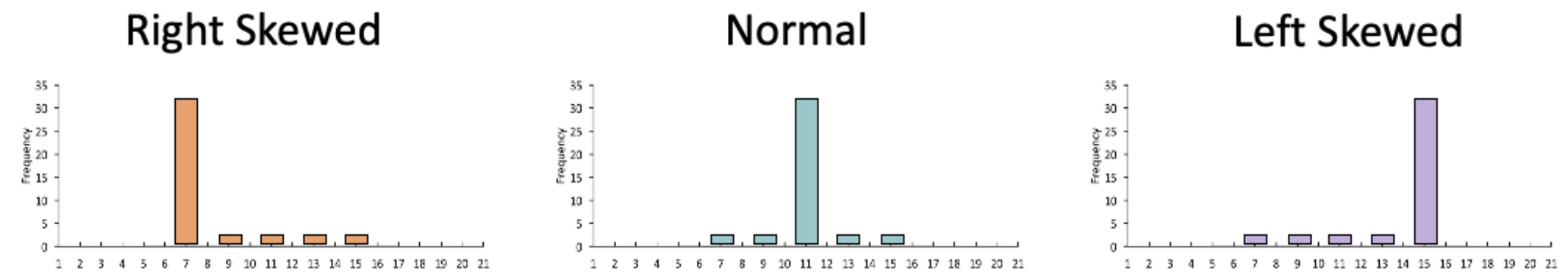

Figure 2. Schematic representations of the stimulus space (panel A) and input distributions (panel B) used in Experiments 1 and 2. Schematic representations of the stimulus space (panel C) and input distributions (panel D) used in Experiment 3. 
In each training trial, participants saw one of the training flowers on the computer screen and had to guess whether it belonged to category A or B. After they selected one of the categories, they saw a feedback sentence "The correct answer is __." After going through the training phase, participants were tested in 21 trials, each containing one of the flowers in the continuum. In each test trial, participants were asked to select which category the flower belonged to. However, unlike the training phase, no feedback was provided in the test phase.

\section{Results}

The main question of interest is whether category representation is affected by the category distribution. Specifically, we asked whether a skewed input distribution, as opposed to a normal distribution of items, during study influenced how broadly participants extended the learned category to new items. To do so, we started by transforming the participants' responses during the test phase so that the category learned with a flat distribution was always on the right and the one with the unimodal distribution was on the left, and such that responses on the left side corresponded to category A and on the left to category B (Fig. 3). Therefore, the right-skewed distribution had the peak on the most extreme left side of the continuum whereas the left-skewed distribution had the peak closer to the center of the continuum (and thus closer to the other training category). This step was important so that participants and conditions could be directly compared.

A secondary question is whether accuracy at classifying studied items (which have an objective, supervised, category assignment as ground truth), differed across conditions and whether it is related to the learner's category broadness. By hypothesis, performance classifying 
studied items at test should be related to generalization broadness because broader categories are likely to include the studied items.

The plot on the top panel of Figure 3 shows the proportion of " $A$ " responses in each condition during the test phase. As it can be seen in the figure, the type of distribution changes how the categories are represented. Specifically, participants who saw a skewed distribution of the category items during training had a wider representation of the category compared to those who trained with normally distributed sample of items.

To quantify the observed impact of different distributions on participants' representations of the categories, we estimated the furthest item in the continuum participants are still likely to accept as a member of category A, i.e., the category change-point. This is a measure of how broad the category is. To estimate the change-point, we identified, for each participant, the highest value classified as A and the lowest value classified as B. The mean-point between these two values approximates the boundary between the two categories for that participant. In the statistical analyses below we included the side in which the skewed distribution was studied (1-6 vs. 17-21) and the stimuli set as covariates. All analyses were followed-up with two planned contrasts to (1) identify the effect of skewness by comparing the average of both skewed distributions vs. normal distributions, and (2) identify the effect of type of skew, by comparing left-skewed vs. right-skewed distributions. 

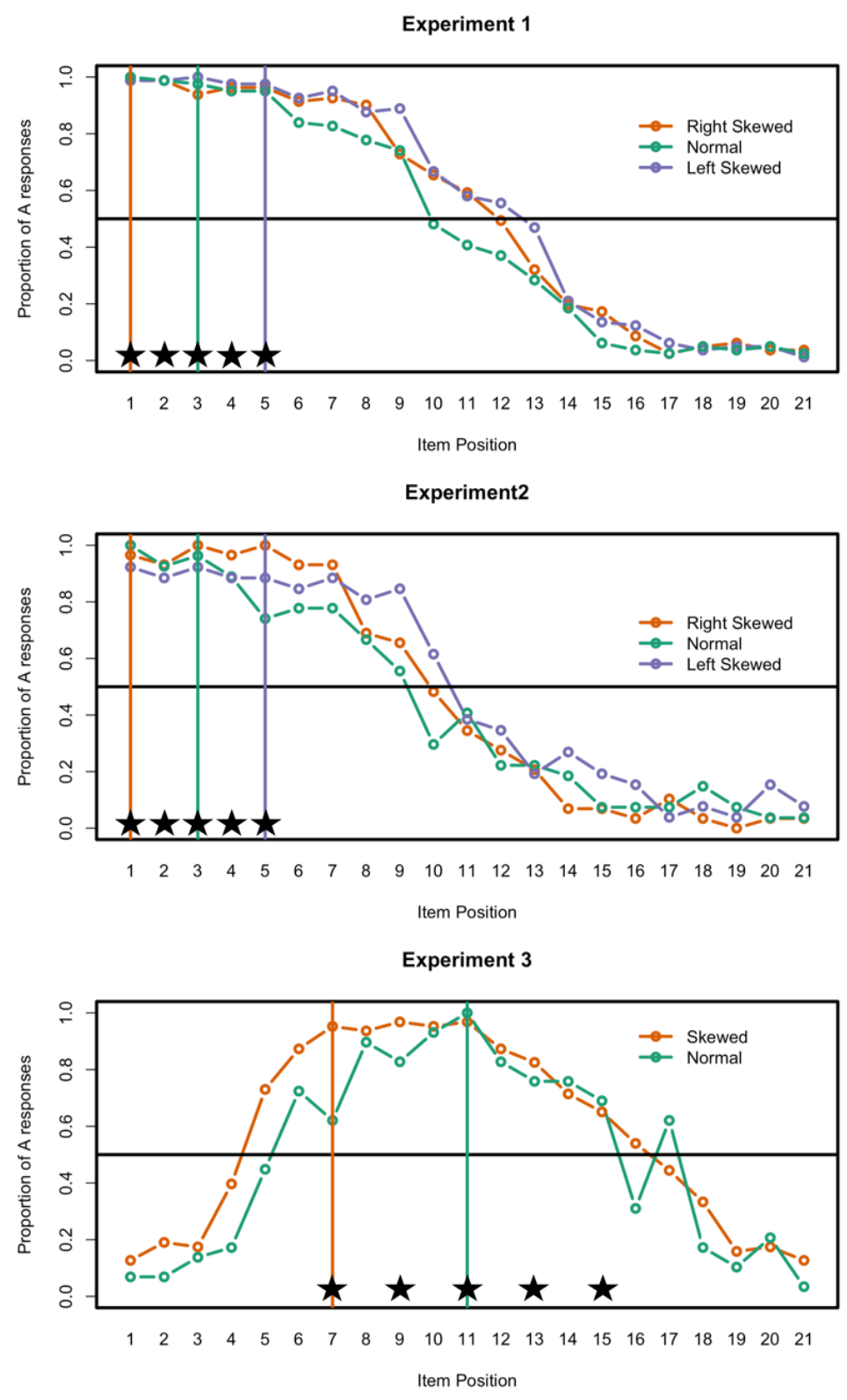

Figure 3. Proportion of "A" responses during test for each experiment (each row from top to bottom: Experiment 1, Experiment 2, and Experiment 3) and study distribution condition. Vertical lines indicate the peak item for the study distribution in each condition, the horizontal line indicates $50 \% \mathrm{~A}$ responses, and the stars indicate studied items. 
How far participants generalized the categories varied across conditions (see Table 1), $F(2,156)=7.05, p=.001, \eta_{G}^{2}=0.05$. Planned contrasts comparing the effect of skewed vs. normal distributions showed that participants extended their category further when a skewed distribution was used during study, $t(158)=3.43, p=.002$. Participants also created slightly broader categories after experiencing a left-skewed distribution of items during study compared to a right-skewed distribution, although this effect was only marginally statistically significant, $t(158)=1.88, p=.062$. One possible reason for this difference is that the item that was seen more frequently is closer to the other category in the left-skewed than the right-skewed category.

Table 1: Mean change point for each condition in Experiments 1 and 2.

\begin{tabular}{lccccc}
\hline & Measure & Right-Skewed & Normal & Left-Skewed & $F$ \\
\hline \multirow{2}{*}{ Exp. 1} & Change point & 11.26 & 10.58 & 11.96 & $7.05^{* *}$ \\
& & $(10.61-11.91)$ & $(9.98-11.19)$ & $(11.51-12.41)$ & \\
\multirow{2}{*}{ Exp. 2} & Change point & 10.03 & 9.51 & 11.25 & $4.45^{*}$ \\
\hline
\end{tabular}

Note: Signif. codes: $0^{\text {‘*** }} 0.001^{\text {‘ } * *} 0.01^{\text {‘*’ }} 0.05^{\prime}+{ }^{\prime} 0.1^{\prime}$ ' $1.95 \%$ confidence intervals in parenthesis.

Finally, we also investigated participants' accuracy at classifying studied items during the test phase. Overall, participants were highly accurate at classifying studied items $(M=0.97,95 \%$ CI: 0.96-0.98), and their accuracy did not differ between conditions, $F(2,156)<1, \eta_{G}^{2}=0.001$. We also correlated participant's change point with their accuracy categorizing studied items to test the hypothesis that broader generalization would be related to better performance 
categorizing studied items at test. This correlation was low $(r=.09)$ and did not reach statistical significance $(p=.0158)$, although this is likely the result of the lack of variability given the high overall accuracy classifying studied items.

\section{Experiment 2}

Overall, the results of Experiment 1 suggest that the distribution of category items during study has an impact on the category representation acquired: skewed distributions seem to promote the creation of broader categories that extend to a wider range of potential new objects.

The main goal of Experiment 2 was to replicate and extend the results of Experiment 1 to skewed distributions with different properties. Specifically, we manipulated the steepness of the skewed distributions by changing the ratio between the most frequent item and the "tail" items. In Experiment 1, we used distributions with a 40:5 steepness ratio, that is, the most frequent item was presented 40 times whereas the remaining four items were presented 5 times each. This makes the more frequent item 8 times more frequent than the remaining items. It is possible that this mild steepness ratio influenced the results and different results would be found if we used more extreme steepness ratios. For example, it is possible that with larger steepness ratios this effect would not be seen, which would lead to the conclusion that the effect observed in Experiment 1 is tied to mild steepness, i.e., flatter distributions. Conversely, if the effect is due to skewness itself, then even if we increase the ratio we should see the same effects. Therefore, in Experiment 2, we compared two different steepness ratios: 28:3 and 32:2.

Although the two steepness ratios are equated for the total number of exposures to the category ( 40 presentations of each category), this number differs from that of Experiment 1 (60 presentations of each category). There were two reasons for this change: (1) it made the task 
shorter, which allowed us to collect data online and expand the population from the undergraduate sample used in Experiment 1, and (2) it allowed us to guarantee that the effect found in Experiment 1 is not tied to the total number of exposures.

Experiment 2 maintains the main manipulation of Experiment 1: using each of the steepness conditions we created three distribution conditions: right-skewed, normal, and left-skewed distributions, by manipulating whether the peak was on the left, center, or right of the sample (as in Experiment 1). Additionally, this manipulation was introduced as a between-subject design in order to reduce the possibility of the mutual influence of each condition on the others.

In sum, the changes introduced in Experiment 2 allow us to test the generalizability of the findings in Experiment 1 to different steepness ratios, the total number of category exposures, different populations, and different experimental setups (from within-subject design to a between-subject design). We predict that, if the results of Experiment 1 are due to the skewness of the categories, then we should find in Experiment 2 that the steepness ratio of the distributions does not affect how learners extend their categories, whereas the distribution of these repetitions will, as we saw in Experiment 1.

\section{Method}

Participants. Using G*Power we determined that the sample size required to detect an effect size of $\eta_{G}^{2}=0.05(\mathrm{f}=0.31)$ as in Experiment 1 in a between-subject design with $75 \%$ power was approximately 75 participants. We recruited 82 adults via Amazon Mechanical Turk (37 females, mean age: 32 ) to participate in this study (we exceeded our goal because we anticipated some dropout). The entire study took approximately 5 minutes and participants were 
paid $\$ .60$ for their participation. Participants were randomly assigned to one of 6 conditions, crossing the distribution steepness ratio (28:3 and 32:2) and the shape of the input distribution (right-skewed, normal, and left-skewed). Recruitment and experimental procedures were approved by Indiana University's Institutional Review Board and all participants gave informed consent prior to participation.

Procedure. Two steepness ratio conditions were used, 28:3 and 32:2 condition. In both conditions, the training procedure was the same as that of Experiment 1, except that each category was trained 40 times.

In the $28: 3$ condition, each item in the uniform condition occurred 8 times. The peak item in the unimodal condition occurred 28 times whereas the rest of the training items occurred 3 times each. There were 3 distribution conditions: right-skewed, normal, and left-skewed distribution.

Similarly, in the 32:2 condition, each item in the uniform condition occurred 8 times. The peak item in the unimodal condition occurred 32 times whereas the rest of the training items occurred 2 times each. Again, there were 3 distribution conditions: right-skewed, normal, and left-skewed distribution.

\section{Results}

We used the same general analytic approach as in Experiment 1. First, we looked at the effect of the steepness of the distribution on how broadly participants generalized the categories using the change-point measure. The effect of steepness was not statistically significant, $F(1,76)=2.32, p=.132, \eta_{G}^{2}=0.03$, or interacted with the other variables, $F<1$. Because there 
was no effect of steepness, we analyzed both steepness conditions together in the remainder of the analyses.

The plot in the middle panel of Figure 3 shows the proportion of " $A$ " responses during the test phase (combining both steepness conditions). Analyses of the extent of generalization (see Table 1) also show a similar pattern to Experiment 1. Overall, the distribution of the category during study influenced the category representation, $F(1,76)=4.45, p=.015, \eta_{G}^{2}=0.10$. Participants extended their categorization further with skewed when compared to normal distributions, $t(79)=2.28, p=.025$, and further with the left-skewed when compared to the right-skewed distribution, $t(76)=2.15, p=.035$.

Next, we analyzed participants' accuracy at classifying studied items during the test phase. As in Experiment 1, participants were highly accurate at classifying studied items ( $M=$ 0.93, 95\% CI: $0.90-0.97)$, and this accuracy did not differ between steepness ratio conditions, $F(1,76)<1, p=.540, \eta_{G}^{2}=0.004$ or between skewness conditions, $F(1,76)=1.01, p=.366, \eta_{G}^{2}=0.026$. Also as in Experiment 1, accuracy at classifying studied items at test did not correlate with our measure of category broadness, $r=-0.131, p=.242$. Although, as before, this is likely the result of a lack of variability in accuracy classifying studied items at test, given participants overall high performance in this task.

\section{Experiment 3}

The results of the previous two studies suggest that the distribution of items during category learning influences the category representation that learners acquire. However, it is 
possible that the existence of a contrasting second category with a uniform distribution influenced the results. Because the uniform distribution was used in one extreme of the space, it was not possible to determine whether the distribution of items during study influences generalization independently from the distribution of the other categories in the space. To extend the results of Experiments 1 and 2, in this third experiment we used a single category and compared the category representations participants created from normal vs. skewed distributions.

\section{Method}

Participants. Using G*Power we determined that the sample size required to detect an effect size of $\eta_{G}^{2}=0.05(\mathrm{~d}=0.62)$ as in Experiment 1 with $75 \%$ power was approximately 84 participants. This sample size was calculated taking into account that one of the groups (Skewed distribution) would have twice as many participants as the other group (Normal distribution), because there are two types of Skewed distributions (left-skewed and right-skewed; See below for details).

92 adults recruited via Amazon Mechanical Turk participated in this study (37 females, mean age: 35). Again, we recruited more participants than required because we anticipated some dropout. The entire study took approximately 5 minutes and participants were paid $\$ .60$ for their participation. Participants were randomly assigned to one of the three conditions. Recruitment and experimental procedures were approved by Indiana University's Institutional Review Board and all participants gave informed consent prior to participation.

Design and Procedure. Like in Experiment 1, the stimuli used in Experiment 3 were 21 flowers. However, instead of having the most extreme items as the training objects, the training 
items in Experiment 3 were items in the middle of the distribution. In addition, to increase the perceptual differences among items, instead of using the 5 items in the middle, we spread out the distance between training items and used items 7, 9, 11, 13, and 15 (Figs. 2C and 2D). Thus, contrary to the two previous experiments, in Experiment 3 in addition to the training items (items $7,9,11,13$, and 15) some novel items were covered by the training category boundary (e.g., items $8,10,12$, and 14) while other novel items were outside of the training boundary (i.e., items more extreme than items 7 or 15).

The members in the training category occurred 40 times in total with a $32: 2$ steepness ratio (as in Experiment 2). One member in the distribution occurred 32 times while the other members occurred 2 times each. There were 3 distribution conditions, right-skewed distribution (i.e., peak at item 7), normal distribution (i.e., peak at item 11), and left-skewed distribution (i.e., peak at item 15).

In each training trial, participants saw one of the training flowers and a label " $\mathrm{A}$ " under it. Participants could take as long as they like before they click a button "next," which starts the presentation of the next trial. Similar to Experiments 1 and 2, after going through the training phase, participants were tested in 21 trials. Participants saw one flower in each test trial and were asked to click whether the flower was an "A" or "Not A" flower. No feedback was provided in the test phase.

\section{Results}

We followed the same analytical approach as in Experiments 1 and 2 with the following exceptions. Because participants could generalize the category towards both the left and the right 
side, we calculated two category change points, one on the left side of the stimuli continuum and one on the right side. When participants classified all items on one side as "A", we estimated the change-point as the maximum possible in the continuum ( 1 or 21 , depending on the side). To determine how broad were the categories that learners acquired, we calculated the difference between the two change points (changepoint right - changepoint left). Finally, as in Experiments 1-2, we merged the left and right-side skewed distributions, by transforming participants responses to map to a single skewed distribution with a peak in item 7 . Because there was no other category learned, right-side and left-side skewed distributions are conceptually the same in this study.

The bottom panel of Figure 3 shows the probability of "A" responses during test for Experiment 3. As can be seen from the figure, the type of distribution (skewed vs. normal), has an impact on how broad the category representation is. Participants extended the category further to the left of the peak when the category is skewed compared to the normally-distributed category (see Table 2), $t(90)=2.74, p=.007, d=0.61$, but not to the right side of the peak, $t(90)=0.82, p=.417, d=0.18$. Finally, participants created a broader category after study with skewed distributions compared to normal distributions, although this difference is small and only marginally significant $t(90)=1.92, p=.058, d=0.43$.

Table 2: Mean change point and broadness of distribution for each condition in Experiment 3. 


\begin{tabular}{cccc}
\hline Measure & Skewed & Normal & $t$ \\
\hline Left-side change point & $5.07(4.69-5.45)$ & $6.05(5.37-6.73)$ & $2.74^{* *}$ \\
Right-side change point & $16.51(15.87-17.15)$ & $16.05(15.15-16.95)$ & 0.82 \\
Broadness & $11.44(10.59-12.28)$ & $10.00(8.76-11.23)$ & $1.92^{+}$
\end{tabular}

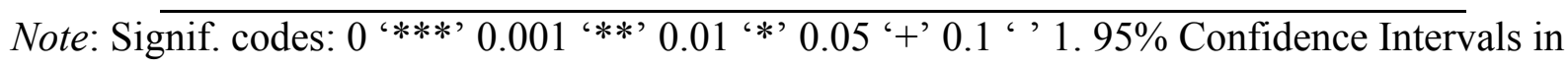
parenthesis.

Again, participants were highly accurate at correctly classifying studied items $(M=0.84$, 95\% CI: 0.81:0.88), though this accuracy is lower than in Experiments 1 and 2. In addition, accuracy at classifying studied items differed between conditions, $t(90)=2.34, p=.021, d=.53$. Participants were more accurate at classifying studied items at test in the Skewed distribution ( $M$ $=0.87,95 \% C I: 0.83: 0.91)$ condition than in the Normal distribution condition $(M=0.78,95 \%$ CI: 0.70:0.87). Finally, participants' accuracy classifying studied items was positively correlated with broadness, $r=.60, p<.0001$.

\section{General Discussion}

Across three studies we investigated the impact that skewed input distributions had on category learning and generalization. The results are straightforward: learning using skewed input distributions results in broader generalization than learning using normal input distributions. This pattern suggests that skewed input distributions might promote broader representation of categories (compared to normal input distributions). 
One possible reason for these results is that people's mental representation of categories is biased towards the mean of the input they received (Duffy et al., 2010; but see Duffy \& Smith, 2018). This proposal is consistent with the findings that for left-skewed distributions for which the mean of the input is closer to the middle of the stimuli space (see Fig. 2B), participants show generalizations biased towards the center of the stimuli space. However, we also find that right-skewed distributions - for which the mean is further away from the center of the stimuli space - result in broader categories as well. Although there is some difference between generalization for left-skewed and right-skewed input distributions, the fact that both generalizations are broader than generalizations resulting from normal input distributions, suggests that this explanation cannot, by itself, account for the entire pattern of results.

We should also note that previous work in category learning has also investigated the related effects of item frequency and item perceptual variability on category learning. For example, Nosofsky (1988) demonstrated that generalization and typicality judgments are biased towards more frequently seen items in the input (see also, Knapp \& Anderson, 1984). However, in this paper, we investigate not only the effect of frequency (the peak of the distribution), but also its position relative to the other studied items. If generalization were pulled towards the most frequent item and its relative position had no effect, then we should see narrower generalizations for the right-skewed distribution than all the other distributions. Instead, we found that both skewed distributions resulted in wider generalization than the normal distribution (for which the peak is equally frequent but located in the middle relative to other studied items). These results suggest that more than item frequency matters for category learning and generalization — the skewness of the distribution itself also plays a role. 
Another possibility is that participants represent the stimuli they experienced as a normal distribution (Flannagan et al., 1986; Huttenlocher et al., 2000) that covers all studied items and is centered around the more frequent item (see Fig. 1A). In essence, if the participants represent the input as a normal distribution, the distance between the most frequent item and the most extreme item of the input is larger in the skewed input distributions than in the normal input distribution (item 1 to item 5 in the right-skewed input distribution vs. items 1 to item 3 or item 5 to item 3 , in the normal input distribution). Consequently, a skewed input distribution is encoded as being wider, resulting in a broader representation of the categories and later classification of more items as belonging to that category. More generally, regardless of the shape of category representation, a long-tail skewed distribution in the training data created a wider category representation than a normal distribution with two short tails.

Yet another possibility is related to how skewness might influence perceived variance of the input. It has been demonstrated that participants create broader categories when the studied items were perceptually more variable (Fried \& Holyoak, 1984; Cohen et al., 2001; Sakamoto et al., 2008). In the present work, we maintained the properties of the items constant in such a way that all conditions had the same training stimuli. However, it is plausible that the distribution affects the perceived variance of the study exemplars. In essence, when the distance between the most frequent item and the last item from the tail is larger (as is the case with skewed distributions), participants perceive the category as more variable (despite that being controlled for). This perceived variation could contribute to broader generalization with skewed distributions without any manipulation of stimuli variation. 
Interestingly, in Experiment 3 we saw that participants' classification of studied items at test differed between the skewed and normal distributions and was correlated with generalization broadness. Although this was not the case in Experiments 1 and 2, this correlation might indicate that different input distributions might influence not only how broad learners generalize their knowledge but also how well they remember studied items and their category assignments. This hypothesis is consistent with findings suggesting that normatively early learned words in infants, tend to refer to objects that have skewed distributions in their daily experience (Clerkin et al., 2017). Future work is necessary to decouple generalization and training performance in order to identify possible cause and effect relationships, that the current work cannot distinguish (i.e., is different learning and memory causing generalization performance or is generalization performance causing different learning and memory for different skew conditions?)

There are two main implications of our findings. First, these results suggest that current categorization theories - which only consider one type of input distribution - might underestimate category generalization and the generalization process. Second, these theories derived from experimental studies using normal distributions of exemplars might be critically misaligned with human category learning in the real world, as most input distributions in the real world are skewed (Boyd \& Goldberg, 2009; Clerkin et al., 2017; Smith et al., 2018; Smith \& Slone, 2017). For example, when children see a skewed input distribution of objects, where there is a clear most frequent item (their favorite sippy cup) but a long tail of substantially less frequent items of the same category (wine glasses; Clerkin et al., 2017), they are creating broad, more inclusive categories. This early process might potentiate early category learning by establishing fewer categories that need to be learned. The same process might also be a potential 
cause for the well-established overgeneralization errors in young children (e.g., Gelman, Croft, $\mathrm{Fu}$, Clausner, \& Gottfried, 1998). By creating broader categories from skewed distributions, children might include as part of the category more distant objects. Consequently, children will not only have fewer categories but also include more distant objects as part of existing categories — thus resulting in overgeneralization.

Finally, the work presented here highlights the importance of studying how information is distributed in the real world. Instead of working on theoretical assumptions about the input, we need to develop experiments, theories, and models that empirically reflect the real world distributions. Because different distributions in the input may engage different learning mechanisms or the same mechanism in different ways, and as a result, create different learning outcomes as demonstrated in the present study, the conclusions we arrive at based on real-world input might differ substantially from the current theories of category learning. 


\section{Acknowledgments}

We are thankful to Nurul Said and Eric Timperman for their help with collecting the data and creating the stimuli. This work was supported in part by NIH grant R01 HD074601 to CY and by Portuguese Science Foundation Graduate Training Fellowship grant \# SFRH/BD/78083/2011 to PC.

Declarations of interest: none. 


\section{Open Practices Statement}

None of the experiments reported in this article was formally preregistered. The data, analysis scripts, and the materials have been made available on a permanent third-party archive:

https://osf.io/xbhgz/ 


\section{Author Contributions}

All authors developed the study concept and contributed to the study design. Testing and data collection were performed by P.F. Carvalho and C. Chen. P.F. Carvalho and C. Chen performed the data analysis and interpretation under the supervision of C. Yu. P.F. Carvalho and C. Chen drafted the manuscript, and $\mathrm{C}$. Yu provided critical revisions. All authors approved the final version of the manuscript for submission. 


\section{References}

Bambach, S., Crandall, D., Smith, L., \& Yu, C. (2018). Toddler-Inspired Visual Object Learning. In Advances in Neural Information Processing Systems (pp. 1209-1218).

Clerkin, E. M., Hart, E., Rehg, J. M., Yu, C., \& Smith, L. B. (2017). Real-world visual statistics and infants' first-learned object names. Philosophical Transactions of the Royal Society B: Biological Sciences, 372(1711), 20160055. https://doi.org/10/gdkz25

Cohen, A. L., Nosofsky, R. M., \& Zaki, S. R. (2001). Category variability, exemplar similarity, and perceptual classification. Memory \& Cognition, 29, 1165-1175.

Duffy, S., Huttenlocher, J., Hedges, L. V., \& Crawford, L. E. (2010). Category effects on stimulus estimation: Shifting and skewed frequency distributions. Psychonomic Bulletin \& Review, 17(2), 224-230.https://doi.org/10/cw7t6z

Duffy, S., \& Smith, J. (2018). Category effects on stimulus estimation: Shifting and skewed frequency distributions-A reexamination. Psychonomic Bulletin \& Review, 25, 1740-1750.

Faul, F., Erdfelder, E., Lang, A.-G., \& Buchner, A. (2007). G*Power 3: A flexible statistical power analysis program for the social, behavioral, and biomedical sciences. Behavior Research Methods, 39, 175-191.

Flannagan, M. J., Fried, L. S., \& Holyoak, K. J. (1986). Distributional expectations and the induction of category structure. Journal of Experimental Psychology: Learning, Memory, and Cognition, 12(2), 241-256. https://doi.org/10/b4gmmn 
Fried, L. S., \& Holyoak, K. J. (1984). Induction of category distributions: A framework for classification learning. Journal of Experimental Psychology: Learning, Memory, \& Cognition, 10, 234-257.

Gelman, S. A., Croft, W., Fu, P., Clausner, T., \& Gottfried, G. (1998). Why is a pomegranate an apple? The role of shape, taxonomic relatedness, and prior lexical knowledge in children's overextensions of apple and dog. Journal of Child Language, 25(2), 267-291.

Goldstone, R. L., Kersten, A., \& Carvalho, P. F. (2017). Categorization and Concepts. In J. Wixted (Ed.) Stevens' Handbook of Experimental Psychology and Cognitive neuroscience, Fourth Edition, Volume Three: Language \& Thought (pp. 275-317) New Jersey: Wiley.

Huttenlocher, J., Hedges, L. V., \& Vevea, J. L. (2000). Why do categories affect stimulus judgment? Journal of Experimental Psychology: General, 129(2), 220-241. https://doi.org/10/d4krhg

Knapp, A. G., \& Anderson, J. A. (1984). Theory of categorization based on distributed memory storage. Journal of Experimental Psychology: Learning, Memory, and Cognition, 10(4), 616.

Kruschke, J. K. (2003). Attention in Learning. Current Directions in Psychological Science, 12(5), 171-175. https://doi.org/10/c8k8jm

Nosofsky, R. M. (1988). Similarity, frequency, and category representations. Journal of Experimental Psychology: Learning, Memory, and Cognition, 14(1), 54.

Rudemo, M. (1982). Empirical choice of histograms and kernel density estimators. Scandinavian Journal of Statistics, 65-78. 
Sakamoto, Y., Jones, M., \& Love, B.C. (2008). Putting the psychology back into psychological models: Mechanistic vs. rational approaches. Memory \& Cognition, 36, 1057-1065.

Smith, L. B., \& Slone, L. K. (2017). A Developmental Approach to Machine Learning? Frontiers in Psychology, 8. https://doi.org/10/gcnzr9

Smith, L. B., Colunga, E., \& Yoshida, H. (2010). Knowledge as process: Contextually-cued attention and early word learning. Cognitive Science, 34(7), 1287-1314. https://doi.org/10.1111/j.1551-6709.2010.01130.x

Smith, L. B., Jayaraman, S., Clerkin, E., \& Yu, C. (2018). The Developing Infant Creates a Curriculum for Statistical Learning. Trends in Cognitive Sciences, 22(4), 325-336. https://doi.org/10/gc9qdr

Werker, J. F., Yeung, H. H., \& Yoshida, K. A. (2012). How Do Infants Become Experts at Native-Speech Perception? Current Directions in Psychological Science, 21(4), 221-226. 